\title{
La fertilización fosforada disminuye la riqueza y aumenta el número de especies exóticas de plantas en pastizales intersembrados con leguminosas
}

\author{
Pedro Pañella ${ }^{1, \varpi}$; Gerónimo Cardozo $;$ Robin Cuadro $;$ Rafael Reyno ${ }^{3}$ \& Felite \\ LEZAMA $^{1}$ \\ ${ }^{1}$ Facultad de Agronomía, Universidad de la República, Montevideo, Uruguay. ${ }^{2}$ Instituto Nacional de Investigación \\ Agropecuaria, Treinta y Tres, Uruguay. ${ }^{3}$ Instituto Nacional de Investigación Agropecuaria, Tacuarembó, Uruguay.
}

\begin{abstract}
Resumen. El Uruguay forma parte de los Pastizales del Río de la Plata, una de las áreas más extensas de pastizal en el mundo, con una biodiversidad destacada. Actualmente, la presión derivada de la intensificación en el uso del suelo determina que estos ecosistemas se encuentren deteriorados. A nivel productivo, una práctica usual es sembrar leguminosas exóticas en cobertura, con fertilización fosforada, sobre pastizal natural. El objetivo de este trabajo fue evaluar el impacto de niveles crecientes de fertilización sobre la riqueza de especies vegetales, la presencia de exóticas y la productividad primaria neta. También se revisaron posibles mecanismos que expliquen los cambios observados en la diversidad. Se establecieron dos sitios experimentales: PA, en las Sierras del Este, y GL, en la Cuesta Basáltica. Se dispuso de ocho tratamientos con niveles variables de fertilización fosforada en parcelas sembradas con Lotus angustissimus. Se calculó la productividad primaria neta total y de L. angustissimus (PPNAt y PPNALa) para los primeros tres años del experimento. Además, se realizó un muestreo florístico al tercer año y medio, en el que se evaluó la riqueza de especies vegetales (total y de exóticas). La riqueza total disminuyó con la fertilización y la riqueza de exóticas aumentó. La PPNAt y la PPNALa aumentaron con la fertilización en PA, pero no en GL. Para PA no es posible afirmar qué mecanismos actúan detrás de la pérdida de especies, mientras que en GL es posible que entren en juego mecanismos de las hipótesis de 'dimensionamiento del nicho' y de 'competencia total'. Concluimos que se debe estudiar más esta tecnología en búsqueda de estrategias de fertilización que logren mejoras en la productividad sin comprometer la biodiversidad.
\end{abstract}

[Palabras clave: fósforo, pastizal natural, riqueza, PPNA, Lotus angustissimus]

Abstract. Phosphorus fertilization lowers richness and increases the number of exotic plant species in grasslands overseeded with legumes. Uruguay sits in the Rio de la Plata Grasslands, one of the biggest grassland biomes in the world, with a pronounced biodiversity. Nowadays, intensification of land use has led to the degradation of this ecosystem. A common practice for enhancing productivity of natural grasslands in the region is the overseeding of an exotic legume, alongside phosphorus fertilization. The objective of this study is to measure the impact of a phosphorous fertilization gradient on total plant species richness, the presence of exotic species and the net primary productivity. With the data obtained, we explored possible mechanisms that explain the changes in diversity observed. Two experimental sites were used: PA (in the geomorphological region 'Sierras del Este'), and GL (in the geomorphological region 'Cuesta Basáltica'). Eight plots were established, with different amounts of phosphorus fertilization, with overseeding of Lotus angustissimus. A vegetation sampling of each treatment was done on the third year and a half after the establishment of the experiment. Total and exotic species richness was evaluated, alongside the effect of each treatment on the total and the $L$. angustissimus net primary productivity of the first three years of the experiment. We found that total species richness decreased with increasing amounts of phosphorus fertilization, while exotic species richness increased with increasing amounts of phosphorus fertilization. The total and the L. angustissimus net primary productivity increased with fertilization for PA, but didn't for GL. This suggests that, in GL, mechanisms regarding the 'niche dimension' and the 'total competition' hypothesis are taking place. In PA, it isn't possible to distinguish which mechanisms are the predominant ones behind the species loss observed. We conclude that more studies need to be done on this technology, in search of fertilization thresholds that allow for greater productivity without putting biodiversity in jeopardy.

[Keywords: natural grassland, species richness, ANPP, phosphorus fertilization, Lotus angustissimus]

Editora asociada: Natalia Perez Harguindeguy $\bar{\triangle}$ ppanella@fcien.edu.uy
Recibido: 4 de Noviembre de 2019

Aceptado: 5 de Junio de 2020 


\section{INTRODUCCIÓN}

Conservar la biodiversidad vegetal requiere conocer los mecanismos que permiten sostenerla. Existen múltiples teorías que intentan explicar la gran cantidad de especies de plantas que coexisten en un mismo hábitat, dado que todas utilizan el mismo conjunto reducido de recursos (Harpole et al. 2016). Una posible explicación la brinda la 'hipótesis del dimensionamiento del nicho' (Harpole et al. 2007), la cual plantea que las comunidades que se ensamblan en torno a la disponibilidad de recursos se encontrarán restringidas de manera estocástica sobre cuán similares pueden ser las especies que coexisten. Las distintas especies tendrán compromisos y compensaciones diferenciales sobre la adquisición de unos recursos sobre los otros por los que compiten, lo que permite así su coexistencia (Harpole et al. 2007). Una adición de nutrientes implicará una disminución en el número de compromisos y favorecerá a algunas especies sobre otras. Por lo tanto, aplicar un fertilizante o incorporar nitrógeno por fijación biológica llevará a un decrecimiento en el número de especies (Harpole et al. 2007). Otras teorías, alternativas y complementarias a la hipótesis del dimensionamiento del nicho también intentan explicar el decrecimiento en el número de especies ante un aumento de nutrientes. La 'hipótesis de la asimetría lumínica' establece que más nutrientes implican incrementos direccionados en la productividad aérea, que llevará a competencia por luz por diferencias de tamaño y sombreado de las especies de mayor porte (Rajaniemi 2002; DeMalach 2018). La 'hipótesis de la competencia total' adhiere a la competencia por luz, pero también supone una mayor productividad subterránea, que lleva a competencia por la captación de nutrientes (DeMalach 2018). Finalmente, la 'hipótesis de la densidad' establece que habrá una mayor productividad aérea que por el sombreado llevará a la pérdida de plántulas, lo que aumenta la mortandad dentro de la comunidad y lleva a la pérdida de especies al azar (Rajaniemi 2002). Existe una amplia evidencia empírica de los problemas que el enriquecimiento de nutrientes (e.g., a través del uso continuo de fertilizantes) puede generar sobre la composición de especies de una comunidad de pastizal, el decrecimiento en riqueza que este manejo puede conllevar, e incluso como esto puede repercutir en un descenso en productividad (Weigelt et al. 2009; Ceulemans et al. 2011, 2013; Harpole et al. 2016). A su vez, se espera que una adición de nutrientes (en particular, si se encuentra ligada a un régimen de disturbios distinto del natural) lleve a una mayor invasibilidad (Huenneke et al. 1990; Alpert et al. 2000; Davis et al. 2000; Fridley et al. 2007).

La biodiversidad de los pastizales naturales tiene gran relevancia, tanto per se como indirectamente por los servicios asociados que presta (Loureau et al. 2001; Weigelt et al. 2009). Desde el punto de vista productivo, en los pastizales se registraron numerosos casos en los que una mayor biodiversidad se liga a un aumento en la productividad (Loureau et al. 2001; Bullock et al. 2007). También existe evidencia para afirmar que una mayor biodiversidad implica una mayor estabilidad frente a fluctuaciones ambientales (la 'hipótesis del seguro' [Yachi and Loureau 1999], de particular importancia), visto que brindaría una estabilidad al forraje frente a los efectos del cambio climático. A su vez, se registró una conexión de la biodiversidad con otras características ecosistémicas beneficiosas, como una menor susceptibilidad al ingreso de especies no deseables a nivel productivo (Weigelt et al. 2009), las cuales tienden a ser nonativas (i.e., exóticas). Las especies no-nativas son aquellas que fueron transportadas a una región al cruzar barreras que previnieron su expansión natural hasta la fecha, como resultado de la actividad humana (Alpert et al. 2000; Richardson et al. 2000). Estas especies podrán naturalizarse, pasar a ser invasoras e incluso transformadoras, si estas generaran efectos negativos sobre los ecosistemas a los que llegan, como la extinción local de las especies nativas. Esta es una de las causas principales de pérdida de especies a nivel mundial (Vitousek et al. 1996; Alpert et al. 2000; Richardson et al. 2000; Fridley et al. 2007). La susceptibilidad de un ambiente a la entrada de nuevas especies, invasibilidad, es una propiedad emergente de los ecosistemas afectados por múltiples factores bióticos y abióticos (Richardson and Pysek 2006). Las barreras bióticas a los procesos de invasión son entendidas bajo el concepto de la resistencia biótica. Incluye todos los efectos negativos de las especies residentes sobre las invasoras, principalmente la competencia por recursos, donde emerge la correlación negativa entre invasibilidad y diversidad (Elton 1958; Alpert et al. 2000).

Los Pastizales del Río de la Plata (PRP) son una región fitogeográfica que ocupa un área de $700000 \mathrm{~km}^{2}$. Comprende la porción noreste de la Argentina, la totalidad del Uruguay y el sur de Brasil, y es una de las áreas más extensas 
del bioma pastizal en el mundo (Soriano et al. 1991). En total, para los PRP se reportan unas 4864 especies nativas de plantas vasculares, pertenecientes a 194 familias y 1324 géneros. Las familias más abundantes son Asteraceae, Poaceae y Fabaceae. La flora del Uruguay cuenta con $\sim 2756$ especies, 126 de las cuales sólo se encuentran en este territorio (Andrade et al. 2018). Bresciano et al. (2014) describen una baja incidencia de especies exóticas para sus pastizales. Actualmente, éstos ocupan un área de 11.5 millones de hectáreas (73\% de la superficie del país) (DICOSE 2017). En efecto, el Uruguay ha sido históricamente un país ganadero, y al presente es una de sus principales actividades económicas (MGAP 2017). Hoy día, grandes extensiones de suelo que solían estar bajo pastizal se usan con fines agrícolas o forestales, reflejo de un cambio acelerado en el uso del suelo (Baeza et al. 2019). Simultáneamente, ha ocurrido un proceso de intensificación de la ganadería. Un ejemplo de esto es la generalización de la práctica denominada 'siembra en cobertura', que en la actualidad abarca al menos $4 \%$ del territorio nacional (DICOSE 2017). Esta estrategia es utilizada para eliminar las restricciones de producción en calidad y cantidad de forraje del pastizal natural asociada sobre todo a limitaciones de $\mathrm{P}$ y N. Esto se logra al sembrar leguminosas exóticas (fijadoras de nitrógeno) en un área, sin antes remover la cobertura vegetal natural del pastizal, y adicionando, además, fertilizantes fosforados (Jaurena et al. 2016). En adelante lo llamaremos pastizal natural con leguminosas y fertilización fosforada (PN-LP). Jaurena et al. (2016) encontraron que en los manejos de PN-LP durante diez años con dos regímenes de fertilización (medio y elevado), la productividad aumentó y se mantuvo alta, pero la diversidad descendió y el número de exóticas aumenta con respecto al pastizal natural.

El objetivo general de este trabajo fue estudiar cómo afecta la fertilización fosforada y la incorporación de leguminosas en la riqueza de especies (totales y exóticas) y en la productividad primaria neta aérea en dos áreas de pastizal de Uruguay. Asimismo, se explora qué mecanismos explican los cambios en la riqueza de especies según las distintas hipótesis de pérdida de la diversidad discutidas anteriormente (Rajaniemi 2002; Harpole et al. 2007; DeMalach 2018). Se espera que la riqueza de especies total descienda. A su vez, se espera encontrar un aumento en el número de especies exóticas al aumentar la fertilización fosforada. Por último, se espera que la productividad primaria neta aérea aumente acorde con el aumento en la fertilización fosforada, y que esta permita vislumbrar distintos mecanismos de pérdida de diversidad. Para alcanzar estos objetivos, se estudiaron dos sitios con características de suelo y vegetación distintas, uno de productividad primaria neta alta y la otra baja, ambos representativos de distintas regiones eminentemente ganaderas del país.

\section{Materiales y Métodos}

\section{Sitios de estudio}

El Uruguay tiene una precipitación media de 1098 mm/año en el sur hasta 1639 mm/año en el norte, con temperaturas medias anuales de $16.5^{\circ} \mathrm{C}$ en el sur y $18.1^{\circ} \mathrm{C}$ en el norte (Dirección Nacional de Meteorología, datos medidos entre 1961 y 1990). La subregión Campos de los PRP comprende al Uruguay en su totalidad (Soriano et al. 1991), y presenta variaciones de vegetación asociadas a la regionalización geomorfológica del país (Panario 1988; Lezama et al. 2019). Este trabajo se ubicó en dos regiones predominantemente ganaderas, las Sierras del Este y la Cuesta Basáltica. Se estableció un sitio experimental por región (Figura 1): uno, en las Sierras del Este, en el sitio experimental de Pasturas y Forraje de INIA, ubicado cerca de la ciudad de Pan de Azúcar (de ahora en adelante, PA), en el Departamento de Maldonado (3446'26"' S 55 ${ }^{\circ}$ '47' O); el otro, en la Cuesta Basáltica, en la Unidad Experimental INIA-Glencoe (GL), en el Departamento de Paysandú $\left(32^{\circ} 0^{\prime} 26^{\prime \prime}\right.$ $\mathrm{S}-57^{\circ} 8^{\prime} 2^{\prime \prime} \mathrm{O}$ ). El sitio PA se caracteriza por presentar suelos medianamente profundos a superficiales, de pendiente leve a moderada. El material geológico está compuesto por rocas ígneas, metamórficas y algunas rocas efusivas ácidas. Se midió su concentración de fósforo en los primeros $15 \mathrm{~cm}$ de perfil previo al experimento, que era por $\mathrm{P}$ (Bray) $2.5 \mu \mathrm{g} \mathrm{P} / \mathrm{g}$ de suelo. El tipo de pastizal se corresponde a la subcomunidad de pastizales ralos IIc Stenachaenium campestre-Andropogon ternatus (Lezama et al. 2019). El sitio GL se caracteriza por presentar suelos profundos, con una pendiente nula. El material geológico está constituido por rocas basálticas. Para los primeros $15 \mathrm{~cm}$ de su perfil, presentó por $\mathrm{P}$ (Bray) $2.1 \mu \mathrm{g} \mathrm{P} / \mathrm{g}$ de suelo. Corresponde a la subcomunidad de pastizales densos IIIb Ruellia morongii-Steinchisma hians (Lezama et al. 2019). 


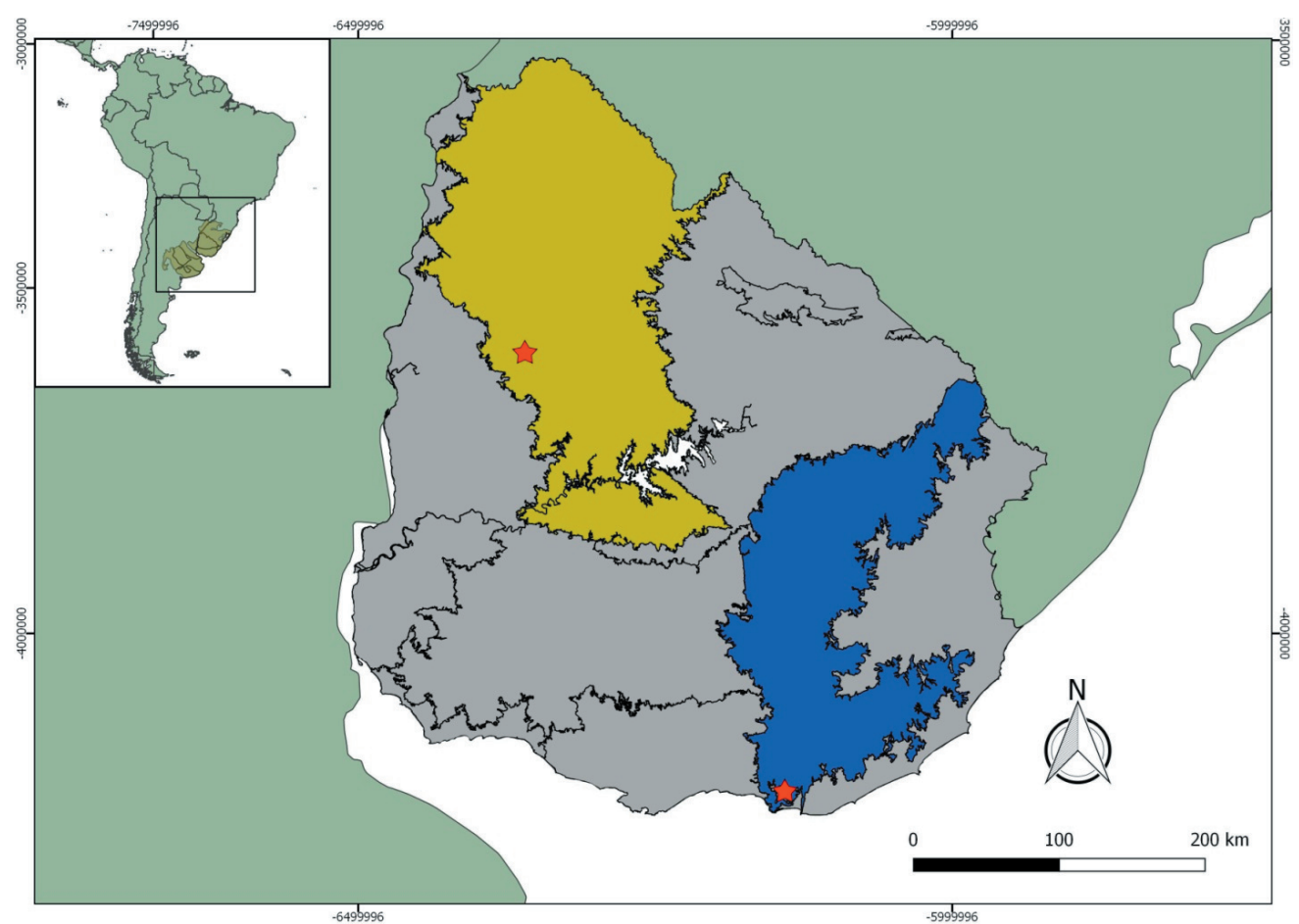

Figura 1. Regiones geomorfológicas del Uruguay, en el contexto de los Pastizales del Río de la Plata (PRP). En amarillo, la Cuesta Basáltica, con una estrella roja marcando la Unidad Experimental Glencoe (GL). En azul, las Sierras del Este, con una estrella roja marcando el sitio experimental de Pasturas y Forrajes (PA). Figure 1. Geomorphological regions of Uruguay, in the context of the Río de la Plata Grasslands (PRP). In yellow, the Cuesta Basáltica, with a red star pointing to the Experimental Unit Glencoe (GL). In blue, the Sierras del Este, with a red star pointing to the experimental site of Pastures and Fodder (PA).

\section{Diseño experimental}

A través de un diseño de bloques al azar se evaluaron los factores siembra de leguminosa y nivel de fósforo agregado, combinado en ocho tratamientos distintos, con tres repeticiones cada uno. En cada sitio se dispusieron 24 parcelas de $6 \mathrm{~m}^{2}$ agrupadas en tres bloques, sin acceso del ganado. Estas se encontraban separadas entre sí por pasillos de $0.5 \mathrm{~m}$ de ancho cubiertos de vegetación. La leguminosa utilizada fue el Lotus angustissimus L. cv. INIA Basalto (Registro Nacional de Cultivares, INASE, $N^{\circ}$ 2019025), un cultivar de ciclo anual invernal seleccionado por su alto desempeño agronómico (Ayala et al. 2017). La siembra fue realizada a una densidad de $5 \mathrm{~kg}$ de semilla/ha en abril de 2014. Se aplicaron seis modalidades diferentes de fertilización fosforada otoñal $\left(\mathrm{P}_{2} \mathrm{O}_{5}\right)$, con una cantidad

Tabla 1. Parcelas experimentales con sus distintos tratamientos de siembra con Lotus angustissimus y fertilizante fosforado. Se incluye cantidad de $\mathrm{P}_{2} \mathrm{O}_{5}$ potencialmente acumulado en los distintos tratamientos a lo largo de los cuatro eventos de fertilización.

Table 1. Experimental plots with their corresponding treatments of sowing with Lotus angustissimus and phosphorus fertilization. Also, the amounts of $\mathrm{P}_{2} \mathrm{O}_{5}$ potentially accumulated in each plot during the four fertilization events.

\begin{tabular}{llcccc}
\hline Código & Tratamiento & $\begin{array}{c}\mathrm{P}_{2} \mathrm{O}_{5} 1^{\circ} \text { año } \\
(\mathrm{kg} / \mathrm{ha})\end{array}$ & $\begin{array}{c}\mathrm{P}_{2} \mathrm{O}_{5} \text { siguientes } \\
\text { años }(\mathrm{kg} / \mathrm{ha})\end{array}$ & $\begin{array}{c}\text { Pac al 30 año } \\
(\mathrm{kg} / \mathrm{ha})\end{array}$ & $\begin{array}{c}\text { Pac al } 4^{\circ} \text { año } \\
(\mathrm{kg} / \mathrm{ha})\end{array}$ \\
\hline La 0/0 & Pastizal con L. angustissimus & 0 & 0 & 0 & 0 \\
La 30/0 & Pastizal con L. angustissimus & 30 & 0 & 30 & 30 \\
La 30/45 & Pastizal con L. angustissimus & 30 & 45 & 120 & 165 \\
La 60/0 & Pastizal con L. angustissimus & 60 & 0 & 60 & 60 \\
La 60/45 & Pastizal con L. angustissimus & 60 & 45 & 150 & 195 \\
La 120/0 & Pastizal con L. angustissimus & 120 & 0 & 120 & 120 \\
PN 0/0 & Pastizal Natural & 0 & 0 & 0 & 0 \\
PN 60/45 & Pastizal Natural & 60 & 45 & 150 & 195 \\
\hline
\end{tabular}


distinta utilizada a la siembra y en las tres subsiguientes refertilizaciones (Tabla 1). Existen dos tratamientos control, uno de pastizal natural sin siembra de L. angustissimus y sin fertilizar, y otro de pastizal natural sin siembra de L. angustissimus, con fertilización inicial y refertilización. Debido a que se registraron altas coberturas de L. angustissimus para este último tratamiento en $\mathrm{PA}$, se tomó a estas parcelas como contaminadas y tanto estas parcelas como su contraparte en GL fueron descartados de los análisis. El régimen diferencial de fertilización llevó a que los distintos tratamientos acumularan distintas dosis de fósforo a lo largo del experimento, de los cuales resaltaremos la acumulación de tres fertilizaciones (en adelante Pac3, al tercer año del experimento) y de cuatro fertilizaciones (en adelante Pac4, al cuarto año), dado que se corresponden con los momentos de toma de datos. Las parcelas estuvieron sujetas a tres o cuatro cortes al año, definido en función de la altura del canopeo (promedio de $15 \mathrm{~cm}$ de altura). El corte se realizó a un remanente de $2 \mathrm{~cm}$ de vegetación, y el material cosechado no fue reintegrado a las parcelas.

Se realizaron muestreos florísticos al tercer año y medio de comenzado el experimento (correspondiente con dosis de fertilizante de Pac4), en un área de $1 \mathrm{~m}^{2}$ en el centro de las parcelas, el cual fue definido como la unidad muestral. En ésta se identificó y asignó valores de abundancia-cobertura a todas las especies vegetales presentes (Dengler et al. 2008). Se cuantificó la riqueza total (S) y de exóticas (Sex) de las unidades muestrales, las cuales se evaluaron mediante ANOVA, seguido de una prueba de comparación múltiple de Tukey y por una regresión lineal de riqueza contra Pac4, trabajando en ambos casos con los logaritmos naturales de las riquezas ( $\mathrm{LnS}$ y LnSex) con el fin de homogeneizar la varianza de las muestras.

Al mismo tiempo, bajo el protocolo de t'Mannetje (2000) se valió de submuestras de los cortes de las parcelas para estimar la productividad primaria neta aérea total (PPNAt) y de L. angustissimus (PPNALa) de los primeros tres años, los cuales fueron analizados por medio de ANOVA, seguido de una prueba de comparación múltiple de Tukey. Los valores de productividad del primer y segundo año (PPNAt1, PPNALa1, PPNAt2 y PPNALa2, correspondiendo los números a los distintos años) fueron utilizados para conocer el estado del pastizal y la historia previa al censo florístico. Además, la productividad del tercer año de comenzado el experimento (mayo de 2016 a marzo de 2017; PPNAt3 y PPNALa3) fue contrapuesta con la dosis de fertilizante acumulado al tercer año (Pac3). Se eligió este año por su mayor correlación con la diversidad medida, visto que era el año más próximo a la fecha de muestreo florístico. Para los análisis estadísticos se utilizó el programa GraphPad Prism 6.0.

\section{Resultados}

\section{Riqueza de especies}

Se registró un total acumulado de 150 especies (15 exóticas) en PA y 109 (12 exóticas) en GL. No se encontraron diferencias significativas en el número de especies por tratamiento (promedio de unidad muestral de $1 \mathrm{~m}^{2}$ de los tres bloques) (Figura 2a) para PA o GL $(F=1.902, P=0.1508$ para $P A ; F=0.9266, P=0.5054$ para GL), siendo esto en promedio $55 \pm 6 \mathrm{y}$ $34 \pm 4$, respectivamente. En cuanto al número de especies exóticas (Figura 2b), se observan diferencias significativas en $\mathrm{PA}$, pero no así en $G L(F=3.954, P=0.016$ para PA; $F=1.432$ y $P=0.2706$ para GL). PA muestra diferencias significativas entre su tratamiento con un mayor número de exóticas, 'La 60/45', con $7 \pm 2$ especies en promedio (13 en total), y su tratamiento con números menores, 'La $0 / 0$ ', con $3 \pm 1$ especies ( 6 en total). En GL había un promedio de $3 \pm 1$ especies exóticas por tratamiento. Los análisis de regresión lineal entre Pac4 y LnS (Figura 2c) mostraron una relación lineal negativa significativa (LnSPA, $P=0.0081, r^{2}=0.3154 ;$ LnSGL, $P=0.0391$, $\left.r^{2}=0.2053\right)$, donde existe una relación inversamente proporcional entre el número de especies y el fósforo acumulado. En cambio, las regresiones lineales de Pac4 y LnSex (Figura 2d) mostraron una relación lineal positiva significativa (LnSexPA, $P=0.0002$, 6, $\mathrm{r}^{2}=0,5339$; LnSexGL, $P=0.01, \mathrm{r}^{2}=0,3013$ ), atestiguando una relación proporcional entre el número de especies exóticas y el fósforo acumulado. En ambos casos, las pendientes son más pronunciadas en PA que en GL (Figura 2c y 2d).

\section{Productividad Primaria Neta Aérea}

La PPNA total del primer año (PPNAt1) y la productividad de L. angustissimus (PPNALa1) en PA mostraron diferencias significativas $(F=4.44, P=0.0101$ y $F=5.73$, $P=0.0034$ respectivamente) (Figura 3). Ambas 


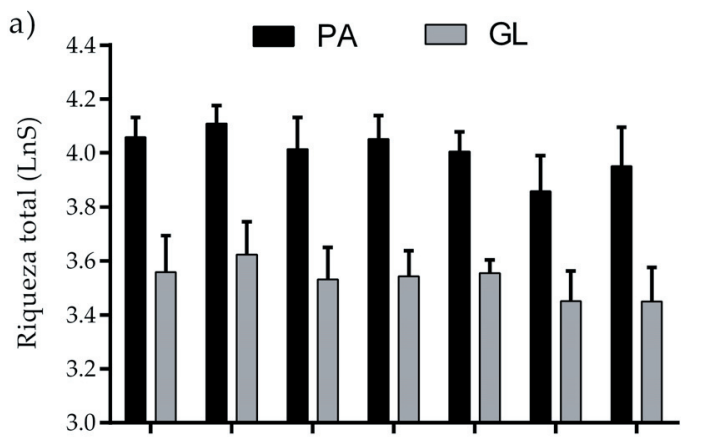

b)

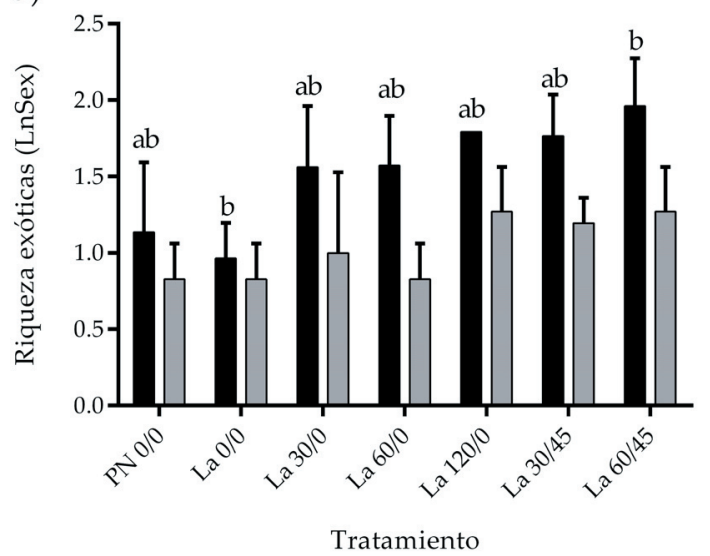

c)

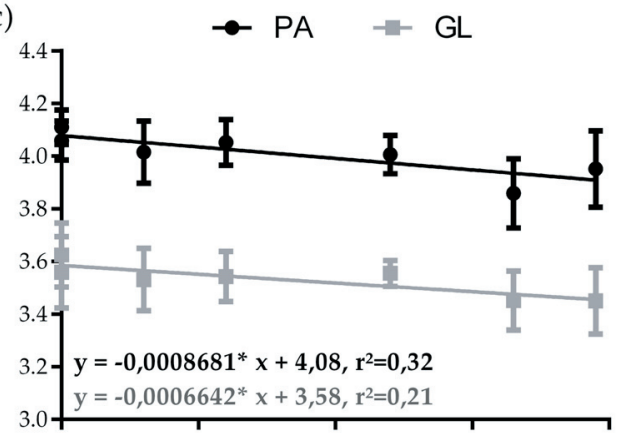

d)

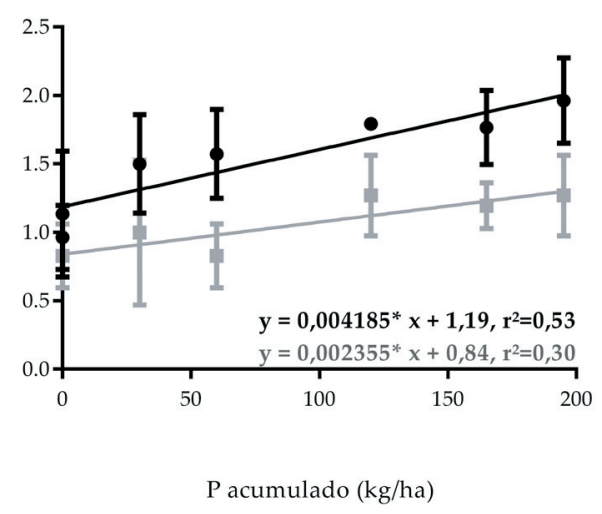

Figura 2. Efecto de los distintos tratamientos sobre: a) el logaritmo de la riqueza total de especies (LnS) y b) el logaritmo de la riqueza de especies exóticas (LnSex). Se señalan con letras diferentes los tratamientos que muestran diferencias significativas para LnSex de PA $(P=0.016)$. Regresión lineal del efecto de la acumulación de cuatro instancias de fertilización fosforada diferencial (Pac4) por tratamiento sobre: c) LnS; d) LnSex. PA: Pan de Azúcar; GL: Glencoe.

Figure 2. Effect of the different treatments on the: a) logarithm of the total species richness (LnS); b) logarithm of the exotic species richness (LnSex). Different letters represent significant differences between treatments for LnSex in PA $(P=0.016)$. Linear regression of the effect of the accumulation of four instances of differential phosphorus fertilization (Pac4) of each treatment on c) LnSt; d) LnSex. PA: Pan de Azúcar; GL: Glencoe.

son máximas en el tratamiento 'La 120/0' y mínimas en el tratamiento 'PN 0/0', acorde con la fertilización. En GL no se encontraron diferencias significativas para la PPNAt1 o PPNALa1 ( $F=0.66, P=0.6815$ y $F=1.72, P=0.1887$, respectivamente), sin una respuesta clara a la fertilización. La PPNAt2 y PPNALa2 no fueron significativa en PA $(F=1.79, P=0.1740$ y $F=1.5, P=0.2471$, respectivamente) ni en $G L$ $(F=0.8, P=0.5868$ y $F=1.24, P=0.3430$ ) (Figura $3)$, aunque se observa una tendencia a una mayor productividad de L. angustissimus en los sitios que recibieron refertilización en PA. GL no muestra una respuesta clara a la refertilización, y tiene una baja PPNALa2.

La PPNA total del tercer año de comenzado el experimento (PPNAt3) (Figura 4a) no dio diferencias significativas para ninguno de los dos sitios $(F=2.165, P=0.1097$ para $P A ; F=0.733$, $P=0.6313$ para $G L)$. Esta fue en promedio 3464 kg.ha-1.año-1 para PA, y $4167 \mathrm{~kg} \cdot$ ha $^{-1}$. año ${ }^{-1}$ para
GL. Por otra parte, se encontraron diferencias significativas para la productividad de $L$. angustissimus del tercer año (PPNALa3) (Figura 4b) en PA, no así en GL $(F=62.65, P<0.0001$ y $F=0.6949, P=0.658$, respectivamente). Para PA, es máxima en el tratamiento 'La 60/45', donde con $1781 \pm 224$ kg.ha- ${ }^{-1}$.año ${ }^{-1}$, representa un 38\% de la PPNAt de ese tratamiento. Es mínima en el tratamiento 'La $0 / 0^{\prime}$, con $0 \pm 0$ kg.ha- ${ }^{-1}$.año ${ }^{-1}$. Para GL, la PPNALa fue en promedio $64 \pm 62$ kg.ha ${ }^{-1}$.año ${ }^{-1}$, lo que representa un $2 \%$ de la PPNAt. Los análisis de regresión lineal entre Pac3 y PPNAt3 (Figura 4c) o PPNALa3 (Figura 4d), mostraron que estos divergían del cero de manera significativas en ambos casos para PA y de manera no significativa en ambos casos para GL. Tanto la PPNAt y la PPNALa de PA se vieron relacionadas positivamente con un aumento en el Pac3 $\left(P=0.0012, \mathrm{r}^{2}=0.4012\right.$ y $P=0.0001, r^{2}=0.748$, respectivamente), de manera más acentuada para la PPNALa (Figuras 4c y 4d). En cambio, la PPNAt y la 
a) $\square \mathrm{PA} \quad \square \mathrm{GL}$

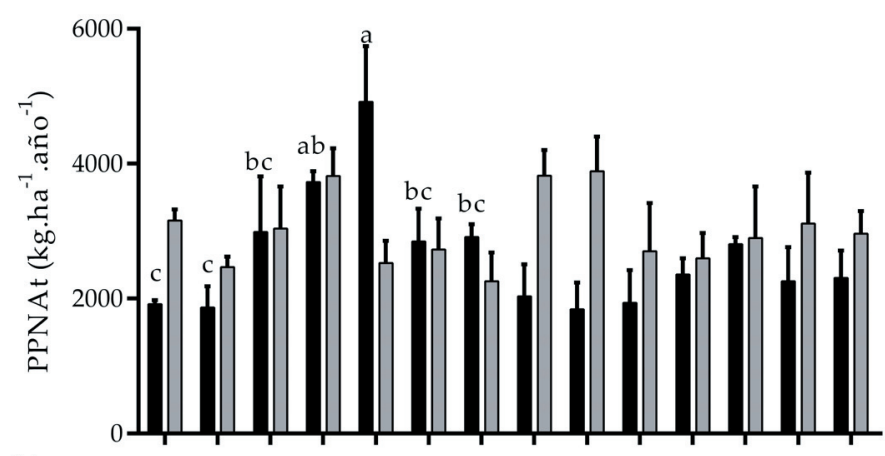

b)

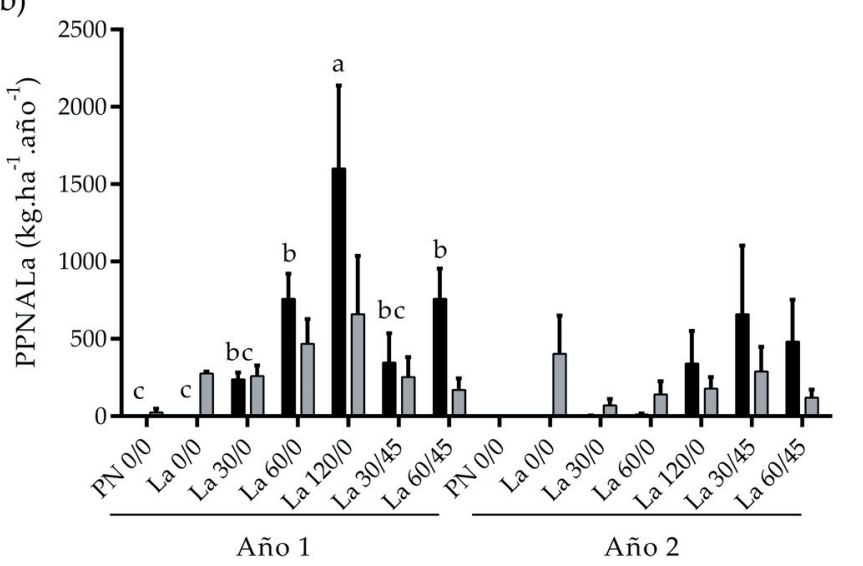

Tratamiento
Figura 3. Efectos de los distintos tratamientos en el Año 1 (2014-2015) y Año 2 (2015-2016) sobre: a) el promedio de la productividad primaria neta aérea total (PPNAt1 y PPNAt2); b) el promedio de la productividad primaria neta aérea de Lotus angustissimus (PPNALa1 y PPNALa2). Se señalan con letras diferentes los tratamientos que muestran diferencias significativas para la PPNAt1 y PPNALa1 en PA ( $P=0.0101$ y $P=0.0034$, respectivamente). PA: Pan de Azúcar; GL: Glencoe.

Figure 3. Effect of the different treatments for Year 1 (2014-2015) and Year 2 (2015-2016) on the averages of: a) the total net primary productivity (PPNAt1 and PPNAt2); b) Lotus angustissimus net primary productivity (PPNALa1 and PPNALa2). Different letters represent significant differences between treatments for PPNAt1 and PPNALa1 in PA ( $P=0.0101$ and $P=0.0034$, respectively). PA: Pan de Azúcar; GL: Glencoe.
PPNALa de GL no se vieron afectadas por el aumento en Pac3 (Figuras 4c y 4d).

\section{Discusión}

\section{Riqueza total y de especies exóticas}

Se observó que, ante un aumento en la fertilización fosforada, en términos de acumulación de entradas de fósforo, decayó la riqueza de especies en ambos sitios estudiados, acorde a nuestra primera predicción. En términos relativos, la respuesta fue similar en ambos sitios, con una pérdida del $20 \%$. Es destacable el hecho de que se observe una respuesta negativa de la riqueza de especies al agregado de $\mathrm{P}$, dado que esto no es lo más usual para los ecosistemas de pastizal, por más que haya algunos antecedentes (Ceulemans et al. 2011, 2013). Generalmente, este tipo de relación entre nutrientes y diversidad en pastizales ha sido observada con el nitrógeno, elemento considerado como 'limitante universal' para la vegetación (Soons et al. 2016). Para nuestro caso, vista la alta tasa de fijación biológica de $\mathrm{N}$ de L. angustissimus, lo más probable es que la mayor cantidad de $\mathrm{P}$ haya permitido una mayor fijación. Por ende, podría ser que se deba al efecto de esta mayor cantidad de $\mathrm{N}$, o al efecto en conjunto de $\mathrm{P}$ y $\mathrm{N}$, lo cual también se encuentra documentado (Harpole et al. 2016).

De acuerdo con la segunda predicción, se encontró que hubo un aumento de las especies exóticas concomitante con un aumento de la fertilización fosforada. Además, se condice con lo observado en el control fertilizado sin siembra de L. angustissimus ('PN 60/ $\left.45^{\prime}\right)$, el cual se vio invadido en PA por esta leguminosa. La pérdida total de especies sólo impacta sobre las nativas, visto que, pese a la pérdida de riqueza total, existe una ganancia de exóticas. Esto fue cierto tanto para PA como GL, aunque fue un cambio de mayor magnitud para el primer sitio, que a su vez fue el que presentó mayor riqueza de especies exóticas en su totalidad. Si se comparan los números de especies exóticas en términos relativos (como una fracción del total de especies), ambos sitios presentan el mismo número de exóticas. Ya se ha documentado 
a)

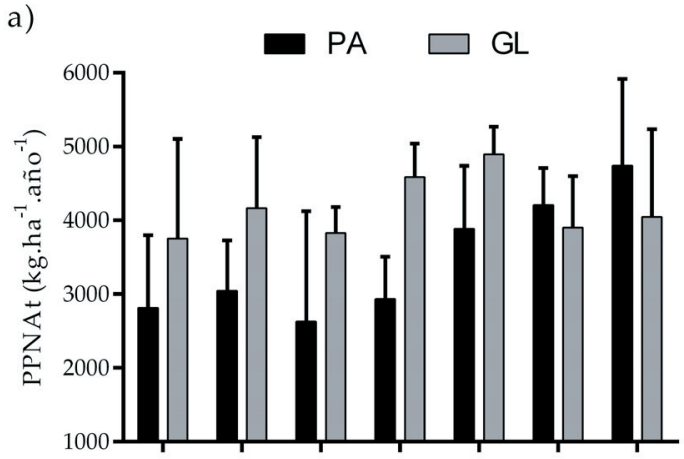

b)

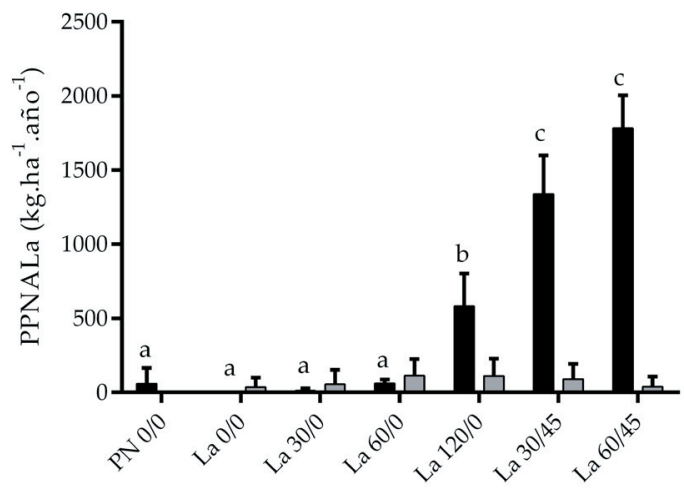

Tratamiento
C)

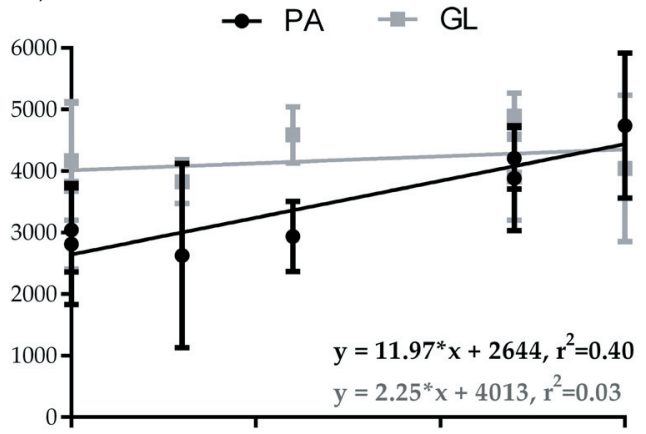

d)

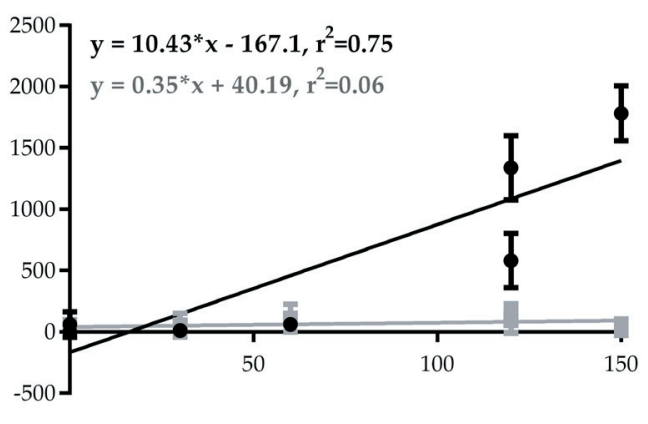

P acumulado (kg/ha)

Figura 4. Efecto de los distintos tratamientos sobre: a) el promedio de la productividad primaria neta aérea total del tercer año (PPNAt3); b) el promedio de la productividad primaria neta aérea de Lotus angustissimus del tercer año (PPNALa3). Se señalan con letras diferentes los tratamientos que muestran diferencias significativas para la PPNALa3 en PA $(P<0.0001)$. Regresión lineal del efecto efecto de la acumulación de tres instancias de fertilización fosforada diferencial (Pac3) por tratamiento sobre la c) PPNAt3; d) PPNALa3. PA: Pan de Azúcar; GL: Glencoe.

Figure 4. Effect of the different treatments on the averages of: a) the total net primary productivity (PPNAt); b) Lotus angustissimus net primary productivity (PPNALa). Different letters represent significant differences between treatments for PPNALa3 in PA $(P<0.0001)$. Linear regression of the effect of the accumulation of three instances of differential phosphorus fertilization (Pac3) for each treatment on: c) PPNAt; d) PPNALa. PA: Pan de Azúcar; GL: Glencoe.

Tabla 2. Hipótesis que explican las causas que llevan a la pérdida de especies ante un aumento de nutrientes en un ecosistema, y la interpretación de cómo explican lo sucedido en los dos sitios de muestreo. PA: Pan de Azúcar; GL: Glencoe.

Table 2. Hypothesis that explain the mechanisms behind species loss when there is a nutrient increase in an ecosystem, and the interpretation of how this hypothesis explain the results observed in the two sites. PA: Pan de Azúcar; GL: Glencoe.

\begin{tabular}{|c|c|c|c|c|}
\hline Teoría & Mecanismo & Observado & Interpretado & Autor \\
\hline $\begin{array}{l}\text { 'Hipótesis del } \\
\text { dimensionamiento } \\
\text { del nicho' }\end{array}$ & $\begin{array}{l}\text { Comunidades ensambladas en } \\
\text { torno a recursos, coexistencia } \\
\text { radica en adquisición diferencial } \\
\text { de nutrientes, desbalance } \\
\text { prioriza a algunas especies. }\end{array}$ & $\begin{array}{l}\text { Aumento de recursos } \\
\text { P y (posiblemente) N } \\
\text { en PA y GL. }\end{array}$ & $\begin{array}{l}\text { Incremento de recursos, } \\
\text { puede implicar exclusión de } \\
\text { peores competidoras en PA } \\
\text { y GL. }\end{array}$ & $\begin{array}{l}\text { Harpole et } \\
\text { al. } 2007\end{array}$ \\
\hline $\begin{array}{l}\text { 'Hipótesis de } \\
\text { la asimetría } \\
\text { lumínica' }\end{array}$ & $\begin{array}{l}\text { Mayor productividad aérea en } \\
\text { algunas especies, competencia } \\
\text { por luz. }\end{array}$ & $\begin{array}{l}\text { Aumento de PPNA } \\
\text { (en particular } L \text {. } \\
\text { angustissimus) por } \\
\text { efecto de fertilización } \\
\text { en PA, no en GL. }\end{array}$ & $\begin{array}{l}\text { Incremento productividad } \\
\text { aérea, puede llevar a } \\
\text { competencia por luz en PA. }\end{array}$ & $\begin{array}{l}\text { Rajaniemi } \\
2002 \\
\text { DeMalach } \\
2018\end{array}$ \\
\hline $\begin{array}{l}\text { 'Hipótesis de la } \\
\text { competencia total' }\end{array}$ & $\begin{array}{l}\text { Mayor productividad aérea y } \\
\text { subterránea en algunas especies, } \\
\text { competencia por luz y por la } \\
\text { captación de nutrientes posterior. }\end{array}$ & & $\begin{array}{l}\text { Incremento productividad } \\
\text { aérea podría incluir } \\
\text { productividad subterránea, } \\
\text { puede llevar a competencia } \\
\text { por luz, agua y nutrientes en } \\
\text { PA. }\end{array}$ & $\begin{array}{l}\text { Rajaniemi } \\
2002 \\
\text { DeMalach } \\
2018\end{array}$ \\
\hline $\begin{array}{l}\text { 'Hipótesis de la } \\
\text { densidad' }\end{array}$ & $\begin{array}{l}\text { Mayor productividad aérea } \\
\text { lleva a mortandad de plántulas, } \\
\text { pérdida azarosa de especies }\end{array}$ & & $\begin{array}{l}\text { Incremento productividad } \\
\text { aérea, puede llevar a pérdida } \\
\text { de plántulas por luz en PA. }\end{array}$ & $\begin{array}{l}\text { Rajaniemi } \\
2002\end{array}$ \\
\hline
\end{tabular}


cambios de este tipo en comunidades similares (Huenneke et al. 1990; Alpert et al. 2000; Davis et al. 2000; Fridley et al. 2007). Esto se podría explicar por la oportunidad de ingreso que genera la pérdida de especies nativas ante el aumento de nutrientes, o por el simple aprovechamiento del pulso de recursos por parte de las especies exóticas, las cuales pueden aprovechar rápidamente la adición de nutrientes 'sobrantes' (Davis et al. 2000).

De forma contraria a lo que se esperaría, dado el concepto más simple de resistencia biótica, el sitio con menor riqueza (GL) fue el que presentó menor incidencia de exóticas. Esto probablemente se deba a que la invasibilidad de un ambiente se explica por una panoplia de características, y noúnicamente por un atributo como podría ser el número de especies, pero es interesante teorizar cuál de estos atributos es el que lo hace particularmente resistente al ingreso de exóticas. El antecedente a nivel de región geomorfológica de Bresciano et al. (2014) no registró diferencias significativas entre la riqueza de especies nativas de ambas regiones, aunque sí de las exóticas (con una menor cantidad de especies exóticas en la Cuesta Basáltica que en las Sierras del Este, al igual que una menor cobertura).

Una explicación posible a la diferencia de especies exóticas entre regiones, se encuentra en un trabajo de Guido et al. (2016). En este se registró que, para la zona de Campos del Norte correspondiente a Brasil, el mayor déficit hídrico (que afectaría de manera adversa a las especies nativas y abriría 'ventanas de invasibilidad'), la mayor densidad de caminería y el incremento en pérdida de pastizal natural en la zona circundante (estas dos últimas implican una mayor cantidad de llegada de propágulos) lleva a un aumento en la aparición de cuatro especies exóticas clave (Ulex europaeus, Eragrostis plana, Cynodon dactylon y Senecio madagascariensis). Esto podría llegar a ser extrapolable para el caso del resto de las especies exóticas no transformadoras. Estas descripciones concuerdan con lo encontrado para nuestros sitios experimentales. PA está ubicado sobre un suelo superficial, al lado de una ruta principal, y en una zona que ha sufrido cambios en la cobertura del suelo en los últimos años, límite con otras zonas geomorfológicas altamente transformadas (Modernel et al. 2016; Baeza et al. 2019), que presentan una mayor incidencia de especies exóticas (Bresciano et al. 2014). En cambio, GL presenta un perfil de suelo profundo (que presenta menor déficit hídrico), se ubica a varios kilómetros de una ruta principal, y se encuentra en una zona poco modificada en cuanto a su cobertura del suelo en los últimos años (Modernel et al. 2016; Baeza et al. 2019). Bajo la óptica del proceso de invasión presentado por Richardson et al. (2000), un menor número de especies logran la introducción y naturalización en GL con respecto a PA. Se necesitarían más estudios y contemplar un mayor número de variables con el fin de lograr respuestas definitivas sobre la dinámica de las especies exóticas en estos pastizales naturales.

\section{Productividad y diversidad}

Se encontraron efectos de los tratamientos sobre la PPNA del tercer año en PA, pero no así en GL. Esto se alinea parcialmente con lo predicho, donde se esperaba un aumento general en la productividad concomitantemente con un aumento en la fertilización. Para el tercer año, la PPNA de GL fue superior a PA en casi todos los casos, a excepción de los tratamientos con alta carga de fósforo, situación en la cual PA logra alcanzar a GL en la PPNA. Las diferencias (o ausencia de diferencias) de la PPNA entre tratamientos $\mathrm{y}$ sitios se encuentran explicadas por la productividad de L. angustissumus, donde en GL presentó una baja PPNA (y por ende no se ve una diferencia clara entre tratamientos), mientras que en PA hay una fuerte presencia de L. angustissimus con una clara respuesta a la fertilización. Los resultados del tercer año se condicen con lo observado en los primeros dos años, donde en PA se registró una mayor PPNA en los tratamientos más fertilizados, y en GL se registró una respuesta poco clara a la fertilización.

A la luz de los efectos de los tratamientos sobre la riqueza total y la PPNA, se revisaron las distintas hipótesis que explican la pérdida de diversidad (resumen en Tabla 2). Debido a que no hubo un aumento consistente en la PPNAt de GL a lo largo del experimento, queda descartada la 'hipótesis de la asimetría de luz' como mecanismo que explica la exclusión de especies, debido que no habría un mayor desarrollo de biomasa que lleve al sombreado en un tratamiento frente a los otros, mientras sí hay una pérdida diferencial de especies (DeMalach 2018). De la misma forma, tampoco se debería esperar un efecto como el predicho por la 'hipótesis de la densidad', dado que al no haber tal sombreado, no habría 
pérdida de plántulas, sea esta aleatoria o direccionada (Rajaniemi 2002). Para PA, no es posible descartar estas dos opciones, dado que el aumento en la fertilización llevó a un aumento en la PPNAt a lo largo de los años, que podría implicar en última instancia un sombreado (DeMalach 2018). Para ninguno de los sitios se puede descartar la 'hipótesis de la competencia total', debido a que no se midió la productividad subterránea (DeMalach 2018). Podría esperarse que, si no existe efecto sobre la PPNAt de GL en ningún año, tampoco lo haya en la productividad primaria neta subterránea (PPNS), visto que se espera que estos flujos estén conectados (Bray 1963; Ni 2004). Sin embargo, existen antecedentes para la región que evidencian que el enriquecimiento en $P$ no necesariamente afectaría la PPNA, mientras sí ejercería un efecto sobre la PPNS (Semmartin et al. 2007). Este podría ser el caso para GL o PA, por lo que no es posible descartar la 'hipótesis de la competencia total'. En aras de dilucidar la importancia de los distintos mecanismos, queda abierto a trabajos posteriores medir los cambios en la PPNS (si los hay) ocasionados por los PN-LP, así como medir la densidad de plantas y la incidencia de la luz por encima y por debajo de la cobertura vegetal, con el fin de ver cuánto de ésta es retenida. Por último, los resultados para ambos sitios podrían respaldar la 'hipótesis del dimensionamiento del nicho' (Harpole et al. 2007), dado que una menor limitación por recursos llevó a una menor riqueza de especies. Ya que no se aislaron los posibles efectos de la PPNAt en PA (por lo que no se puede descartar una opción o la otra), esta podría ser una explicación tan válida como las otras, e incluso podrían complementarse. En cambio, para el caso de GL la pérdida de especies no fue concomitante con una respuesta en productividad aérea, por lo que esta hipótesis es la más plausible para explicar la pérdida en diversidad, sin poder descartar tampoco la 'hipótesis de la competencia total'. Ante un enriquecimiento en nutrientes, las especies con capacidad de aprovechar de mejor manera la nueva estequiometría del suelo compitieron por estos recursos, principalmente $\mathrm{P}$ y $\mathrm{N}, \mathrm{y}$ lograron excluir a las peores competidoras, lo cual disminuye la riqueza total.

\section{Conclusiones e implicancias aplicadas}

Teorizamos que las dinámicas observadas podrían resumirse bajo el siguiente mecanismo: ante un aumento en la disponibilidad de recursos (sea esto de forma directa, por la adición de $\mathrm{P}$, o indirecta, por la fijación biológica de $\mathrm{N}$ ), se pierden especies nativas de la comunidad. Por exclusión competitiva, las especies que se encuentran establecidas en la comunidad bajo esta nueva estequiometría no permiten el reingreso de las especies perdidas. Esta evidencia apoya la existencia de un estado post PN-LP propuesto por Jaurena et al. (2016) de difícil reversión, caracterizado por su baja diversidad y alto grado de invasión por especies exóticas.

Nuestros resultados permiten vislumbrar efectos adversos de la implementación de los PN-LP sobre la diversidad de especies del pastizal natural. Los ecosistemas menos diversos serán también menos resistentes y resilientes (Yachi and Loreau et al. 1999). Esto se refleja directamente en los problemas que trae el cambio climático a la provisión de forraje, los cuales pueden agravarse de tener una comunidad simplificada (Loreau et al. 2001; Jaurena et al. 2016; Modernel et al. 2016). Además, esta simplificación de la comunidad significa un detrimento para la correcta provisión de otros servicios ecosistémicos de interés (Loureau et al. 2001; Weigelt et al. 2009). Más aun, la mayor presencia de especies exóticas puede acentuar estos problemas, directa o indirectamente, como amenaza a la diversidad de especies nativas (Alpert et al. 2000; Fridley et al. 2007). Además, las prácticas que incluyen fertilización tienen otros efectos adversos sobre el ambiente ya que existe un mayor riesgo de escape de fósforo por escorrentía y conduce a la eutrofización de cuerpos de agua (Peterson et al. 2017).

Es pertinente concebir los pastizales como un agroecosistema multifuncional que depende de una alta biodiversidad, por lo que requiere de una conciliación entre la producción y la conservación (Jaurena et al. 2016; Modernel et al. 2016). Esto implica que se debe tener cautela ante la generalización de las prácticas agrícolas del tipo PN-LP con el fin de no comprometer la biodiversidad. Para el caso de la Cuesta Basáltica la tecnología no impactó de manera significativa en la productividad primaria neta aérea del sistema, aunque sí demostraron afectar los parámetros de diversidad evaluados. Por ende, de acuerdo a nuestros resultados sería recomendable explorar más a fondo la implementación de $L$. angustissimus y su funcionamiento en la Cuesta Basáltica a largo plazo previo a su uso en los sistemas agrarios de esta región, visto que, a la luz de nuestros datos, estos meramente significan un deterioro del agroecosistema. Por otro lado, en el sitio que se encuentra en 
las Sierras del Este, la práctica de fertilización con P y siembra de una leguminosa lleva a un aumento en la productividad primaria neta aérea. En este escenario, podrían realizarse nuevos experimentos donde se busque un rango de fertilización menos perjudicial para la biodiversidad y que logre mejoras en el rendimiento del pastizal. De acuerdo a lo visto en nuestros resultados, en niveles cercanos al 'La 60/0' la comunidad no se encuentra particularmente comprometida, y se observa una mejora en productividad (aunqueesta noes demasiado pronunciada). Se podrían explorar niveles cercanos de fertilización, y distintos regímenes de refertilización (e.g., bianuales en lugar de anuales, o de una dosis menor a la contemplada en nuestros tratamientos), en busca de condiciones que logren mantener una comunidad relativamente estable y diversa, con un aumento en la productividad primaria neta aérea beneficiosa para la explotación ganadera. Sin embargo, visto como el L. angustissimus invadió las parcelas fertilizadas y no sembradas (PN 60/45) en este sitio, se debe resaltar el peligro de esta especie como invasora de pastizales enriquecidos en nutrientes.

Agradecimientos. Se agradece al Ing. Agr. Martín Baez y a la Dra. Bianca Andrade por la colaboración durante los eventos de muestreo, y a las Dras. Claudia Rodríguez y Anaclara Guido por la revisión y corrección del manuscrito. Este trabajo contó con el apoyo económico del INIA, el proyecto CSIC I+D 556 y el proyecto Innovagro 148811.

\section{REFERENCIAS}

Alpert, P., E. Bone, and C. Holzapfel. 2000. Invasiveness, invasibility and the role of environmental stress in the spread of non-native plants. Perspectives in Plant Ecology, Evolution and Systematics 3:52-66. https://doi.org/10.1078/14338319-00004.

Andrade, B. O., E. Marchesi, S. Burkart, R. Setubal, F. Lezama, S. Perelman, A. A. Schneider, R. Trevisan, G. E. Overbeck, and I. I. Boldrini. 2018. Vascular plant species richness and distribution in the Río de la Plata grasslands. Botanical Journal of the Linnean Society 188:250-256. https://doi.org/10.1093/botlinnean/boy063.

Ayala, W., M. Bemhaja, F. Condón, B. Cotro, M. J. Cuitiño, J. Docanto, J. García, F. Gutiérrez, F. Olmos, D. Real, M. Rebuffo, R. Reyno, C. Rossi, and J. Silva. 2017. Forrajeras. Catálogo de Cultivares 2017. Instituto Nacional de Investigación Agropecuaria, Montevideo, Uruguay.

Baeza, S., G. Rama, and F. Lezama. 2019. Cartografía de los pastizales naturales en las regiones geomorfológicas de Uruguay predominantemente ganaderas. Ampliación y actualización. Pp. 27-47 en A. Altesor, L. López-Mársico and J. M. Paruelo (eds.). Bases ecológicas y tecnológicas para el manejo de pastizales II. Serie FPTA № 69, INIA, Montevideo, Montevideo, Uruguay.

Bray, J. R. 1963. Root production and the estimation of net productivity. Canadian Journal of Botany 41:65-72. https: //doi.org/10.1139/b63-007.

Bresciano, D., C. Rodríguez, F. Lezama, and A. Altesor. 2014. Patrones de invasión de los pastizales de Uruguay a escala regional. Ecología Austral 24:83-93.

Bullock, J. M., R. F. Pywell, and K. J. Walker. 2007. Long-term enhancement of agricultural production by restoration of biodiversity. Journal of Applied Ecology 44:6-12. https://doi.org/10.1111/j.1365-2664.2006.01252.x.

Ceulemans, T., R. Merckx, M. Hens, and O. Honnay. 2011. A trait-based analysis of the role of phosphorus vs. nitrogen enrichment in plant species loss across North-west European grasslands. Journal of Applied Ecology 48:1155-1163. https://doi.org/10.1111/j.1365-2664.2011.02023.x.

Ceulemans, T., R. Merckx, M. Hens, and O. Honnay. 2013. Plant species loss from European semi-natural grasslands following nutrient enrichment - is it nitrogen or is it phosphorus? Global Ecology and Biogeography 22:73-82. https: //doi.org/10.1111/j.1466-8238.2012.00771.x.

Davis, M. A., J. P. Grime, and K. Thompson. 2000. Fluctuating resources in plant communities: a general theory of invasibility. Journal of Ecology 88:528-534. https://doi.org/10.1046/j.1365-2745.2000.00473.x.

DeMalach, N. 2018. Toward a mechanistic understanding of the effects of nitrogen and phosphorus additions on grassland diversity. Perspectives in Plant Ecology, Evolution and Systematics 32:65-72. https://doi.org/10.1016/ j.ppees.2018.04.003.

Dengler, J., M. Chytry, and J. Ewald. 2008. Phytosociology. Pp. 2767-2779 in S. E. Jorgensen and B. D. Fath (eds.). Encyclopedia of Ecology. Elsevier, Oxford, UK. https://doi.org/10.1016/B978-008045405-4.00533-4.

DICOSE. 2017. Datos de la declaración jurada. Ministerio de Ganadería, Agricultura y Pesca, Montevideo, Montevideo, Uruguay.

Elton, C. S. 1958. The ecology of invasions by animals and plants. Methuen, London, UK. https://doi.org/10.1007/9781-4899-7214-9.

Fridley, J. D., J. J. Stachowicz, S. Naeem, D. F. Sax, E. W. Seabloom, M. D. Smith, T. J. Stohlgren, D. B. Tilman, and B. Von Holle. 2007. The invasion paradox: Reconciling pattern and process in species invasions. Ecology 88:3-17. https: //doi.org/10.1890/0012-9658(2007)88[3:TIPRPA]2.0.CO;2.

Guido, A., E. Vélez-Martin, G. E. Overbeck, and V. D. Pillar. 2016. Landscape structure and climate affect plant invasion in subtropical grasslands. Applied Vegetation Science 19:600-610. https://doi.org/10.1111/avsc.12263. 
Harpole, W. S., and D. Tilman. 2007. Grassland species loss resulting from reduced niche dimension. Nature 446: 791-793.

Harpole, W. S., L. L. Sullivan, E. M. Lind, J. Firn, P. B. Adler, E. T. Borer, J. Chase, P. A. Fay, Y. Hautier, H. Hillebrand, A. S. MacDougall, E. W. Seabloom, R. Williams, J. D. Bakker, M. W. Cadotte, E. J. Chaneton, C. Chu, E. E. Cleland, C. D'Antonio, K. F. Davies, D. S. Gruner, N. Hagenah, K. Kirkman, J. M. Knops, K. J. La Pierre, R. L. McCulley, J. L. Moore, J. W. Morgan, S. M. Prober, A. C. Risch, M. Schuetz, C. J. Stevens, and P. D. Wragg. 2016. Addition of multiple limiting resources reduces grassland diversity. Nature 537:93-96. https://doi.org/10.1038/nature05684.

Huenneke, L. F., S. P. Hamburg, R. Koide, H. A. Mooney, and P. M. Vitousek. 1990. Effects of Soil Resources on Plant Invasion and Community Structure in Californian Serpentine Grassland. Ecology 71:478-491. https://doi.org/10.2307/ 1940302.

Jaurena, M., F. Lezama, L. Salvo, G. Cardozo, W. Ayala, J. Terra, and C. Nabinger. 2016. The Dilemma of Improving Native Grasslands by Overseeding Legumes: Production Intensification or Diversity Conservation. Rangeland Ecology and Management 69:35-42. https://doi.org/10.1016/j.rama.2015.10.006.

Lezama, F., A. Altesor, M. Pereira, and J. M. Paruelo. 2019. Grasslands of Uruguay: classification based on vegetation plots. Phytocoenologia 49(3):211-229. https://doi.org/10.1127/phyto/2019/0215.

Loreau, M., S. Naeem, P. Inchausti, J. Bengtsson, J. P. Grime, A. Hector, D. U. Hooper, M. A. Huston, D. Raffaelli, B. Schmid, D. Tilman, and D. A. Wardle. 2001. Biodiversity and Ecosystem Functioning: Current Knowledge and Future Challenges. Science 294:804-808. https://doi.org/10.1126/science.1064088.

Ministerio de Ganadería, Agricultura y Pesca. 2017. Anuario Estadístico Agropecuario 2017. Dirección de Estadística Agropecuaria, Montevideo, Montevideo, Uruguay.

Modernel, P., W. A. Rossing, M. Corbeels, S. Dogliotti, V. Picasso, and P. Tittonell. 2016. Land use change and ecosystem service provision in Pampas and Campos grasslands of southern South America. Environmental Research Letters 11. https://doi.org/10.1088/1748-9326/11/11/113002.

$\mathrm{Ni}$, J. 2004. Estimating net primary productivity of grasslands from field biomass measurements in temperate northern China. Plant Ecology 174:217-234. https://doi.org/10.1023/B:VEGE.0000049097.85960.10.

Panario, D. 1988. Geomorfología del Uruguay. Propuesta de un marco estructural y un esquema de evolución del modelado del relieve uruguayo. Departamento Publicaciones, Facultad de Humanidades y Ciencias, Universidad de la República, Montevideo, Uruguay.

Peterson, H. M., L. A. Baker, D. Bruening, J. L. Nieber, J. S. Ulrich, and B. N. Wilson. 2017. Agricultural Phosphorus Balance Calculator: A tool for watershed planning. Journal of Soil and Water Conservation 72:395-404. https://doi.org/ 10.2489/jswc.72.4.395.

Rajaniemi, T. K. 2002. Why Does Fertilization Reduce Plant Species Diversity? Testing Three Competition-Based Hypotheses. Journal of Ecology 90:316-324. https://doi.org/10.1046/j.1365-2745.2001.00662.x.

Richardson, D. M., P. Pyšek, M. Rejmánek, M. G. Barbour, F. D. Panetta, and C. J. West. 2000. Naturalization and invasion of alien plants: concepts and definitions. Diversity and Distributions 6:93-107. https://doi.org/10.1046/j.14724642.2000.00083.x.

Richardson, D. M., and P. Pyšek. 2006. Plant invasions: merging the concepts of species invasiveness and community invasibility. Progress in Physical Geography 30:409-431. https://doi.org/10.1191/0309133306pp490pr.

Semmartin, M., M Oyarzabal, J. Loreti, and M. Oesterheld. 2007. Controls of primary productivity and nutrient cycling in a temperate grassland with year-round production. Austral Ecology 32:416-428. https://doi.org/10.1111/j.14429993.2007.01706.x.

Soons, M. B., M. M. Hefting, E. Dorland, L. P. Lamers, C. Versteeg, and R. Bobbink. 2016. Nitrogen effects on plant species richness in herbaceous communities are more widespread and stronger than those of phosphorus. Biological Conservation 212:390-397. https://doi.org/10.1016/j.biocon.2016.12.006.

Soriano, A., R. J. León, O. E. Sala, R. S. Lavado, V. A. Deregibus, M. A. Cahuépé, O. A. Scaglia, C. A. Velázquez, and J. H. Lemcoff. 1991. Rio De Plata Grasslands. Pp. 367-408 en R. T. Coupland (ed.). Ecosystems of the World: Natural Grasslands. Volume 8A. Elsevier, Amsterdam, The Netherlands.

t'Mannetje, L. 2000. Measuring biomass of grassland vegetation. Pp. 151-177 en L. t'Mannetje and R. Jones (eds.). Field and laboratory methods for grassland and animal production research. CABI Publishing, Wallingford, England. https://doi.org/10.1079/9780851993515.0151.

Vitousek, P. M., C. M. D’Antonio, L. L. Loope, and R. Westbrooks. 1996. Biological invasions as global environmental change. American Scientist 84:468-478.

Weigelt, A., W. W. Weisser, N. Buchmann, and M. Scherer-Lorenzen. 2009. Biodiversity for multifunctional grasslands: equal productivity in high-diversity low-input and low-diversity high-input systems. Biogeosciences 6:1695-1706. https://doi.org/10.5194/bg-6-1695-2009.

Yachi, S., and M. Loreau. 1999. Biodiversity and ecosystem productivity in a fluctuating environment: the insurance hypothesis. Proceedings of the National Academy of Sciences 96:463-1468. https://doi.org/10.1073/pnas.96.4.1463. 